\title{
Simultaneous Determination of Registration and Deformation Parameters among 3D Range Images
}

\author{
Tomohito MASUDA, Yuichiro HIROTA, Katsushi IKEUCHI \\ The University of Tokyo \\ 4-6-1 Komaba, Meguro-ku, Tokyo 153-8505, Japan \\ \{tom, hirota, ki\}@cvl.iis.u-tokyo.ac.jp \\ Ko Nishino \\ Columbia University \\ 1214 Amsterdam Avenue, MC 0401 New York, NY 10027, USA \\ kon@cs.columbia.edu
}

\begin{abstract}
Conventional registration algorithms are mostly concerned with rigid-body transformation parameters between a pair of $3 D$ range images. Our proposed framework aims to determine, in a unified manner, not only such rigid transformation parameters but also various deformation parameters, assuming that the deformation we handle here is strictly defined by some parameterized fomulation derived from the deformation mechanism. In this respect, our proposed framework is different from the deformation researched in such fields as medical imaging.

Like other conventional registration algorithms, our algorithm is formulated as a minimization problem of the squared sum of the distance between the corresponding points among a pair of range images. While conventional registration algorithms usually minimize this sum concerned about six parameters (three translation and three rotation parameters), the evaluation function in our proposed algorithm includes deformation parameters as well. Our proposed algorithm can be applied to a wide range of areas of computer vision, in particular, shape modeling and shape analysis. In this paper, we describe how we formulated such an algorithm, implemented it, and evaluated its performance.
\end{abstract}

\section{Introduction}

A 3D data registration algorithm determines the translation and rotation parameters between a pair of the corresponding 3D range images. The algorithm solves the nonlinear equation to minimize the distance between a pair of corresponding 3D range image with respect to the six unknown parameters (three translation and three rotation parameters).

There are many uses for 3D data registration. In research that requires modeling of objects in the real world, it is necessary to have multiple observations of the object in order to cover the whole surface of the object. Aligning these partial 3D data using a registration algorithm is one of the crucial steps to completing the 3D surface model of the object. Registration algorithms are also used to compare differences in shape between similar objects for industrial inspection of manufacturing accuracy. And in archeological applications, there is a need to observe shape deformation, such as the process of deterioration over time. Due to these uses, many registration algorithms have been proposed.

Some applications, however, require determining more parameters than just the six translation and rotation parameters. As a simple example, when comparing 3D data of two objects with the same shape but different size, we have to determine the scaling parameter in addition to the six translation and rotation parameters. This is also the case when aligning data for a deformable object. And when we replace a part of the range data, as we must do for a cylinder, with a CAD primitive model in order to reduce the amount of data or refine its shape, the parameters of the $\mathrm{CAD}$ primitive shape (the diameter and the height in the case of a cylinder) should be determined from the measured data by fitting the primitive to the range data. A conventional registration algorithm cannot solve these problems because it formulates the registration only as a rigid-body transformation.

In this paper, we propose an extended framework of the conventional registration algorithm to overcome these difficulties. This kind of registration, namely, deformation registration, has been researched in such fields as medical imaging [1] [2] [3] [4] [5], where the target object for registration is mainly soft tissue. These researchers adopt similarity, affine, quadric/superquadric, and displacement-field-based transformation so that their deformation works well for any kind of target shape.

These methods can be generally adopted in shape modeling and fitting. However, if the deformation is strictly defined by some parameterized formulation derived from the deformation mechanism, the deformation is much more accurate. The parameters obtained from this strict formulation carry with them the origin of the deformation. So our framework pays as much attention to the parameters obtained in the deformation registration as to the resulting appearance of the deformation. In this respect, our aim is different from those of the researchers cited above. Assuming that changes in shape are strictly represented by a mathematical formula (including some variable parameters) that is known a priori, 
we formulate an extended registration that allows the 3D data to be deformed and determines deformation, translation, and rotation parameters.

The remainder of this paper is organized as follows. Section 2 reviews conventional registration formulas in terms of their robustness. Our proposed formulation need to be robust because our algorithm becomes sensitive to incorrect matching correspondence due to parameter increases. Section 3 describes the design and implementation of the robust rigid-body transformation as a basis of our extended framework. 4 presents how we extend the basic algorithm in order to be able to handle the deformation parameters. Section 5 and 6 describe how to apply our proposed algorithm to actual applications. In particular, we determine the shape parameters of a plaster function model made in the 19th century for mathematical education . Another example of our registration algorithm is to align two range images obtained from a stably setting and floating laser range sensor, referred to as a Floating Laser Range Sensor (FLRS). Due to the floating characteristics, the second sensor causes data distortion. Our algorithm can determine both rigid-body-transformation (translation and rotation) and distortion parameters. We also evaluate the accuracy of this algorithm. Finally, Section 7 summarizes this paper with a conclusion and ideas about future work.

\section{Related Work}

One of the most fundamental algorithms for 3D data registration is the Iterative Closest Point (ICP) algorithm proposed by Besl and McKay [6]. This algorithm framework reduces registration to the problem of minimizing the sum of the distance between the corresponding data by iterative calculation. Function minimization with respect to the transformation parameters leads to the optimal ones that represents the plausible transformation between the data pieces being aligned, for example, three translation and three rotation parameters in the case of rigid-body transformation. This framework is extended in various ways that we can classify as registration ordering, matching unit, and error metric.

\subsection{Registration Odering}

In the registration of multiple sets of 3D data, ordering affects the convergence of the final result. Two types of ordering are considered here: sequential and simultaneous ordering. Sequential ordering selects a corresponding pair of data pieces at each iteration for registration, and repeats this process until all the data pieces are aligned [7] . Its computation cost is low because only two data pieces are handled at each iteration. However, it is susceptible to registration failure since registration errors are locally accumulated, and this causes inaccuracy of the registration result 1 ).

In contrast, simultaneous ordering aligns all the data together at each iteration. Although its computation cost is higher, it enables more accurate registration because the registration error is distributed globally.

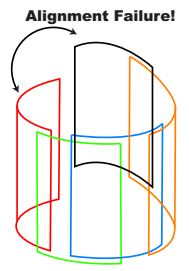

Figure 1. Illustration of registration failure in sequential strategy. The data are sequentially aligned counterclockwise in order from the red data and the black data, and the accumulated error prevented the black and red data from the correct registration.

\subsection{Matching Unit}

The matching unit of the ICP algorithm is of two kinds: (1) the geometric feature points and (2) all the points in the range images. Assuming that one-to-one correspondence is taken among all the feature points, the feature point matching doesn't change its correspondence at any iteration [8] [9] . So it cannot achieve accurate registration if the correspondence cannot be taken precisely. All-points matching updates the correspondence so that it can be more plausible as the iteration proceeds [10] [11], and therefore can achieve more accurate registration, as Rusinkiewicz et. al. reported [12] .

\subsection{Error Metric}

As the error metric, the point-to-point or point-to-plain distance of the matching unit is mainly used [13] [14]. Some other algorithms adopt such additional information as the reflectance (the reflection ratio of the laser ray) and color of the captured point as the error metric [15] .

\section{Robust Determination of Translation and Rotation Parameters}

Our basic registration algorithm is designed to robustly determine translation and rotation parameters as accurately as possible even though we are sacrificing convergence speed. Accurate convergence is a more important factor than rapid convergence because our final objective is to determine deformation parameters in addition to translation and rotation parameters.

The 3D data that is obtained by a laser range sensor has considerable measurement noise. So a normal metric cannot be estimated from the point cloud with acceptable reliability. Therefore, the closest point-to-point distance should be employed as the error metric as described in [12]. To cope with erroneous measurement, simultaneous ordering is adopted.

As explained earlier, our basic registration algorithm aligns the closest points together with all data pieces simultaneously so as to minimize the sum of the point-to-point distance. Minimization of the error function is represented 
as follows:

$$
E(\mathbf{p})=\frac{1}{N(M-1)} \sum_{i}^{N} \sum_{j}^{M} \rho\left(z_{i j}(\mathbf{p})\right),
$$

where

$$
\begin{aligned}
\mathbf{p} & =(\mathbf{t}, \mathbf{q}) \\
z_{i j}(\mathbf{p}) & =\left\|\mathbf{R}(\mathbf{q}) \mathbf{x}_{i}+\mathbf{t}-\mathbf{y}_{j i}\right\|^{2}, \\
\rho\left(z_{i j}(\mathbf{p})\right) & =\log \left(1+\frac{1}{2} z_{i j}(\mathbf{p})^{2}\right),
\end{aligned}
$$

t : : translation vector,

$\mathbf{R}(\mathbf{q})$ : rotation matrix corresponding to quaternion $q$,

$\mathbf{x}_{i} \quad$ : $i$ th point in the data set of interest,

$\mathbf{y}_{j i} \quad$ : the corresponding point of $x_{i}$,

in the $j$ th measured data,

$\begin{array}{ll}N & : \quad \text { the number of data points, } \\ M & : \quad \text { the number of measured data }\end{array}$

The range images are aligned iteratively by moving (translating/rotating) the measured data according to the estimated parameters. The movement is determined such that the total sum of the distance between the corresponding points is minimized. As for the rotation matrix, we use the quaternion representation of three degree of freedom. Finally, we solve the six dimensional vector $\mathbf{p}$ in order to minimize the sum of $z_{i j}(\mathbf{p})$ for all $i, j$.

In the direct square sum error function, considerable noise leads to the imprecise registration of $3 \mathrm{D}$ data because the exact correspondences between the noisy data in the initial step are unavailable. The erroneous correspondences must be removed before registration. In this algorithm, M-estimation is used for noise elimination (Function 4 ) by considering the probability distribution of the error. Lorentz function is used here since it yields the best result as written in [16] .

On this error metric $E(\mathbf{p})$, we compute the parameters $\mathbf{p}$ which fulfill the following equation:

$$
\mathbf{p}_{\text {opt }}=\arg \min _{\mathbf{p}} E(\mathbf{p}) \text {. }
$$

For the gradient-based solution of the non-linear optimization, the descent gradient is computed as follows:

$$
\begin{aligned}
\frac{\partial E}{\partial \mathbf{p}} & =\frac{1}{N(M-1)} \sum_{i}^{N} \sum_{j}^{M} \frac{\partial \rho\left(z_{i j}\right)}{\partial z_{i j}} \cdot \frac{\partial z_{i j}}{\partial \mathbf{p}} \\
& =\frac{1}{N(M-1)} \sum_{i}^{N} \sum_{j}^{M} w\left(z_{i j}\right) z_{i j} \frac{\partial z_{i j}}{\partial \mathbf{p}}, \\
& \text { where } w\left(z_{i j}\right)=\frac{1}{z_{i j}} \cdot \frac{\partial \rho\left(z_{i j}\right)}{\partial z_{i j}} .
\end{aligned}
$$

If we evaluate $\partial z_{i j} / \partial \mathbf{p}$ by identifying quaternion $q_{I}$, we can represent $\partial z_{i j} / \partial \mathbf{p}$ as

$$
\begin{aligned}
\frac{\partial z_{i j}(\mathbf{p})}{\partial \mathbf{p}} & =\left.2\left(\mathbf{R}(\mathbf{q}) \mathbf{x}_{i}+\mathbf{t}-\mathbf{y}_{j i}\right) \frac{\partial\left(\mathbf{R}(\mathbf{q}) \mathbf{x}_{i}+\mathbf{t}-\mathbf{y}_{j i}\right)}{\partial \mathbf{p}}\right|_{\mathbf{q}_{I}} \\
& =\left[\begin{array}{c}
2\left(\mathbf{x}_{i}+\mathbf{t}-\mathbf{y}_{j i}\right) \\
-4 \mathbf{x}_{i} \times\left(\mathbf{t}-\mathbf{y}_{j i}\right)
\end{array}\right]
\end{aligned}
$$

\section{Simultaneous Determination of Deforma- tion Parameters}

Our proposal assume that deformation can be represented by some parameterised mathematical formula, and is known a priori, but that its parameters are unknown.

Our goal is to simultaneously determine these deformation, translation, and rotation parameters by comparing the target data to transform with its corresponding data. The translation and rotation parameters are determined in a minimization paradigm described in the previous section. If we fix these parameters, the determination of the deformation parameters becomes a shape-matching problem at an iterative minimization step. Thus, we can handle parameter determination in a unified minimization framework.

We extend the parameter estimation of the registration formulation to add the shape parameter by extending the error function in Equation ( 3 ). Therefore, $z_{i j}(\mathbf{p})$ in Equation ( 3 ) is transformed into:

$$
\begin{array}{ll}
z_{i j}(\mathbf{p})=\sum_{i, j}\left\|\mathbf{R}(\mathbf{q}) \mathbf{g}\left(\mathbf{x}_{i}, \mathbf{k}\right)+\mathbf{t}-\mathbf{y}_{j i}\right\|^{2}, \\
\text { where } \begin{array}{ll}
\mathbf{p}= & (\mathbf{t}, \mathbf{q}, \mathbf{k}), \\
\mathbf{g}\left(\mathbf{x}_{i}, \mathbf{k}\right): & \begin{array}{l}
\text { deformation function of point } \mathbf{x}_{i} \\
\text { with respect to parameter } \mathbf{k} .
\end{array}
\end{array}
\end{array}
$$

And the gradient described in Equation ( 7 ) is extended as:

$$
\begin{aligned}
\frac{\partial z_{i j}(\mathbf{p})}{\partial \mathbf{p}}= & 2\left(\mathbf{R}(\mathbf{q}) \mathbf{g}\left(\mathbf{x}_{i}, \mathbf{k}\right)+\mathbf{t}-\mathbf{y}_{j i}\right) \frac{\partial\left(\mathbf{R}(\mathbf{q}) \mathbf{g}\left(\mathbf{x}_{i}, \mathbf{k}\right)+\mathbf{t}-\mathbf{y}_{j i}\right)}{\partial \mathbf{p}} \\
& =\left[\begin{array}{c}
2\left(\mathbf{g}\left(\mathbf{x}_{i}, \mathbf{k}\right)+\mathbf{t}-\mathbf{y}_{j i}\right) \\
-4 \mathbf{g}\left(\mathbf{x}_{i}, \mathbf{k}\right) \times\left(\mathbf{t}-\mathbf{y}_{j i}\right) \\
2\left(\mathbf{g}\left(\mathbf{x}_{i}, \mathbf{k}\right)+\mathbf{t}-\mathbf{y}_{j i}\right) \frac{\partial\left(\mathbf{g}\left(\mathbf{x}_{i}, \mathbf{k}\right)\right)}{\partial \mathbf{k}}
\end{array}\right]
\end{aligned}
$$

This straightforward extension causes unstable convergence of the deformation registration because the obtained translation, rotation, and deformation parameters overreaches its optimum if every parameter is applied simultaneously to the deformation, though each parameter can be estimated with enough accuracy if it is applied independently. Every parameter interferes every other parameter.

In order to prevent this interference, we designed our extended formulation again to remove the translation and rotation effect caused only by deformation. The basic idea is to recover the position and posture that change due to the deformation. This is implemented by "preliminary" rigid-body transformation, which determines only the deformation parameters. First, every parameter is acquired by Equation ( 6 ) and ( 9 ) Then the preliminary rigid-body transformation is determined only by the deformation parameter as follows:

$$
\mathbf{g}^{\prime}\left(\mathbf{x}_{i}, \mathbf{k}\right)=\mathbf{R}_{o} \mathbf{g}\left(\mathbf{x}_{i}, \mathbf{k}\right)+\mathbf{t}_{o}
$$

where

$$
\left(\mathbf{R}_{o}, \mathbf{t}_{o}\right)=\left(\mathbf{R}\left(\mathbf{q}_{o}\right), \mathbf{t}_{o}\right)
$$

such that

$$
\left(\mathbf{q}_{o}, \mathbf{t}_{o}\right)=\arg \min _{\mathbf{q}, \mathbf{t}} \sum_{i}^{N}\left\|\mathbf{R}(\mathbf{q}) \mathbf{g}\left(\mathbf{x}_{i}, \mathbf{k}\right)+\mathbf{t}-\mathbf{x}_{i}\right\|^{2} .
$$


$\mathbf{R}_{o}, \mathbf{t}_{o}$ can be derived from the following equation:

$$
\frac{\partial \sum_{i}^{N} \epsilon_{i}^{2}}{\partial \mathbf{t}_{o}}=\sum_{i}^{N} 2 \epsilon_{i} \cdot \frac{\partial \epsilon_{i}}{\partial \mathbf{t}_{o}}=\mathbf{0},
$$

where

$$
\epsilon_{i}=\mathbf{g}\left(\mathbf{x}_{i}, \mathbf{k}\right)+\mathbf{t}_{o}-\mathbf{x}_{i}
$$

This is a conventional registration problem, but it is unnecessary to strictly solve the above equation. In fact, $\mathbf{R}_{o}$ doesn't affect stable convergence so much as $\mathbf{t}_{o}$. If $\mathbf{R}_{o}$ is ignored, $\mathbf{t}_{o}$ in Equation ( 12 ) is concretely derived as follows:

$$
\begin{array}{r}
\quad \sum_{i}^{N}\left(\mathbf{g}\left(\mathbf{x}_{i}, \mathbf{k}\right)+\mathbf{t}-\mathbf{x}_{i}\right)=\mathbf{0} \\
\therefore \mathbf{t}_{o}=-\frac{\sum_{i}^{N}\left(\mathbf{g}\left(\mathbf{x}_{i}, \mathbf{k}\right)-\mathbf{x}_{i}\right)}{N} .
\end{array}
$$

Finally, Equation ( 8 ) is replaced with:

$$
z_{i j}(\mathbf{p})=\sum_{i, j}\left\|\mathbf{R}(\mathbf{q})\left\{\mathbf{R}_{o} \mathbf{g}\left(\mathbf{x}_{i}, \mathbf{k}\right)+\mathbf{t}_{o}\right\}+\mathbf{t}-\mathbf{y}_{j i}\right\|^{2} .
$$

In the ICP based registration algorithm, the acquisition of a good initial parameter is significant for the optimal registration result. In our implementation, the initial transformation parameter is set manually by GUI with accuracy enough to reach the optimum. We investigate the registration behavior according to the difference between the optimum and the initial setting parameter in each experiment after this section.

\section{Unknown Parameter Estimation of Mathematical Model}

As one of our experiments, we examined the manufacturing accuracy of mathematical models made of plaster (Figure 2 -(1)). This model is a kind of cultural asset. It was manufactured in Germany at the end of the 19th century for educational purposes and has been exhibited in our university museum. This model visually represents the following mathematical formula:

$$
X(u, v)=(l \phi(v) \cos u, l \phi(v) \sin u, l \psi(v)),
$$

$$
\begin{aligned}
& \text { where } \\
& \begin{array}{c}
0 \leq u \leq 2 \pi, \\
-a \cdot \sinh ^{-1}\left(\frac{a}{b}\right) \leq v \leq a \cdot \sinh ^{-1}\left(\frac{a}{b}\right), \\
\phi(v)=b \cosh \left(\frac{v}{a}\right), \\
\psi(v)=\int_{0}^{v} \sqrt{1-\frac{b^{2}}{a^{2}} \sinh ^{-1}\left(\frac{t}{a}\right)} d t .
\end{array}
\end{aligned}
$$

This surface is generated by rotating a 2D catenary (Figure 2 -(2)) according to the related documentation. The surface by revolution always has the azimuthal symmetry. Besides scale parameter $(l), 2$ parameters $(a, b)$ are involved in the deformation of the revolutional surfaces. Because there was no documentation to identify the three deformation parameters when manufacturing the model, our purpose here was to estimate the deformation parameter by applying our proposed framework to its range image and the data computed from Equation ( 15 ), to evaluate the manufacturing accuracy

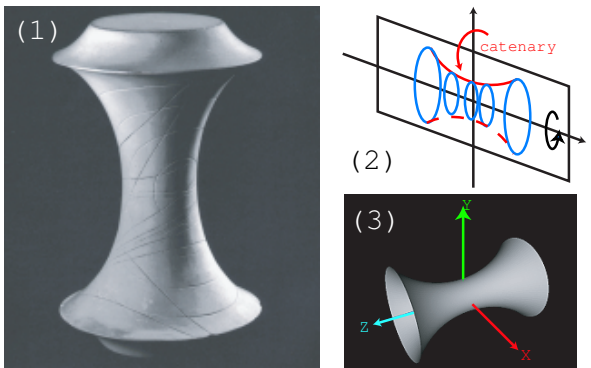

Figure 2. Mathematical model used in our experiment.

of the plaster model under the estimated parameter, and to remake an accurate model to satisfy the interest of historiana and mathematicians in the manufacturer's skill in those days.

We have to estimate these parameters in order to compare the range image of the model and the computed data from Equation ( 15 ) under the estimated parameters. In this case, our proposed method can be applied by replacing the deformation function like this:

$$
\begin{aligned}
\mathbf{g}\left(\mathbf{x}_{i}, \mathbf{k}\right)= & \left(l \phi\left(v_{i}\right) \cos u_{i}, l \phi\left(v_{i}\right) \sin u_{i}, l \psi\left(v_{i}\right)\right), \\
\text { where } & \mathbf{k}=(l, a, b) .
\end{aligned}
$$

The descent gradient is in the following:

$$
\frac{\partial z_{i j}(\mathbf{p})}{\partial \mathbf{p}}=\left[\begin{array}{c}
2\left(\mathbf{g}\left(\mathbf{x}_{i}, \mathbf{k}\right)+\mathbf{t}-\mathbf{y}_{j i}\right) \\
-4 \mathbf{g}\left(\mathbf{x}_{i}, \mathbf{k}\right) \times\left(\mathbf{t}-\mathbf{y}_{j i}\right) \\
2\left(\mathbf{g}\left(\mathbf{x}_{i}, \mathbf{k}\right)+\mathbf{t}-\mathbf{y}_{j i}\right) \frac{\partial \mathbf{g}\left(\mathbf{x}_{i}, \mathbf{k}\right)}{\partial \mathbf{k}}
\end{array}\right],
$$

where

$$
\frac{\partial\left(\mathbf{g}\left(\mathbf{x}_{i}, \mathbf{k}\right)\right)}{\partial \mathbf{v}}=\left[\begin{array}{c}
\left((l \cos u) \frac{\partial \phi}{\partial a},(l \sin u) \frac{\partial \phi}{\partial a}, l \frac{\partial \psi}{\partial a}\right)^{T} \\
\left((l \cos u) \frac{\partial \phi}{\partial b},(l \sin u) \frac{\partial \phi}{\partial b}, l \frac{\partial \psi}{\partial b}\right)^{T} \\
\left(\phi\left(v_{i}\right) \cos u_{i}, \phi\left(v_{i}\right) \sin u_{i}, \psi\left(v_{i}\right)\right)^{T}
\end{array}\right] .
$$

Such that

$$
\begin{aligned}
\frac{\partial \phi}{\partial a} & =-\frac{b v}{a^{2}} \sinh \frac{v}{a} \\
\frac{\partial \psi}{\partial a} & =\frac{v b^{2}}{2 a^{3}}\left(-\frac{1}{2 a^{2}} \sinh ^{-1} \frac{v}{a}+\frac{1}{\sqrt{v^{2}+a^{2}}}\right) \\
\frac{\partial \phi}{\partial b} & =\cosh \frac{v}{a} \\
\frac{\partial \psi}{\partial b} & =-\frac{b}{a^{2}} \sinh ^{-1} \frac{v}{a} .
\end{aligned}
$$

\subsection{Experiment}

The 3D shape of the model was captured by the MINOLTA VIVID 900 . The data was initially aligned by the manual process via GUI. The initial shape parameters were also manually estimated. Figure 3 shows the registration process. It indicates that the computed data is transformed to fit the actual one. Estimated parameter is: $a=0.0568, \quad b=$ $0.0237, \quad l=0.996$. 


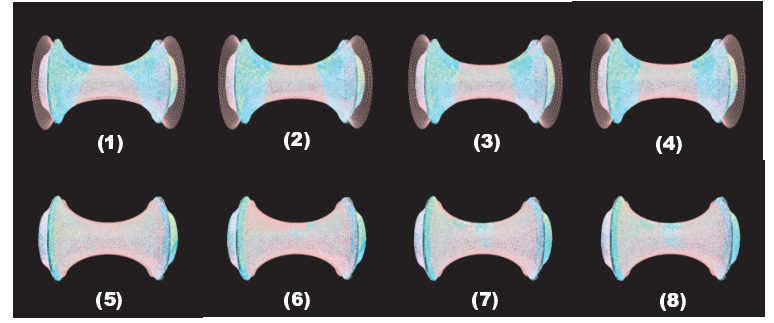

Figure 3. The registration process of the parametric data. (1)-(8) shows the convergence of both the measured and computed data.

\subsection{Evaluation}

The estimation is affected by various kinds of errors: measurement error; initial registration error; and error in an initially input parameter. So we investigated how the accuracy of an estimated parameter depends on such errors by using the synthesized data that was computed under the known parameters with Gaussian noise added for forming the noisy data. The accuracy of the estimation could be evaluated as the difference between the known parameter (ground truth) and the obtained parameters. The deformation parameters were set as $a=0.05, b=0.02$ and $l=1.00$.

The first error to consider in the estimation was the measurement error of the range sensor. We first investigated how much noise was caused according to the pose of the actual object by using the measured data of the white Lambertian plane at different poses. The system is shown in Figure 4

The white board was set on the turntable. The pose was changed by rotating and translating the turntable. We denote $l$ and $\theta$ by the distance from the white board to the laser range finder and the angle between the normal of white board to the ray of the laser, respectively. Five sets of data were obtained at each pose. Principal component analysis (PCA) was applied to estimate the most plausible plane composed by the point cloud and to obtain the standard deviation of the measurement error of the plane data. The standard deviation changes of the plane data are shown in Figure 5.

We investigated the influence of the measurement noise by using the computed data from Equation ( 15 ) and its synthesized data. Noise with different standard deviation was added to its computed data. Standard deviations were set to 0.01 , $0.1,1.0,10.0$ in this experiment. Ten sets of synthesized data were created for each standard deviation as the synthesized data. The initial translation, rotation, and deformation parameters of the synthesized data were the same as those of the computed data. The red line in Figure 6 show the range of the maximum and minimum of estimated parameters $a$, $b$ and $l$, respectively, the blue line shows the average of the estimated parameters, and the green dotted line is the ground truth.

Effects of the noise standard deviation to the estimated parameters were similar in all parameters: the larger the standard deviation was, the greater the difference was between the estimated and the ground truth, and the larger the range of the maximum and minimum parameters was. However, noise added in this experiment was far higher than the ob- served noise. Even though noise with a standard deviation of 0.01 was added, the difference from the ground truth was almost zero, and the parameters were stably estimated. The maximum standard deviation of the measurement noise in MINOLTA VIVID 900 was detected at less than 0.002 , so the result indicated the robustness of our estimation method against the sensor noise.

The next error was the initial registration error of translation and rotation. In the same manner, we added the noise to the computed data to create its corresponding synthesized data. The standard deviation of the noise $(\sigma)$ was set to 0.0004 according to the measurement error as observed above. Each initial parameter was set to the same value as the computed data. The coordinate of the model is shown in Figure 2 -(3).

Effects of translation and rotation were investigated separately. For the initial translation, synthesized data were translated $0.01,0.02$ and $0.03[\mathrm{~m}]$ along $\mathrm{x}$ or $\mathrm{z}$ axis, respectively. For the initial rotation, three sets of synthesized data were rotated 10,20 and 30 [degree] around $x$ axis. Since revolutional surface of catenary has the $\mathrm{x}, \mathrm{y}$ and $\mathrm{z}$ symmetry, these translation and rotation were sufficient for the evaluation.

The results of estimation were shown in Figure 7 . In the figure, the left, middle and right graphs show the estimation result in the case where the synthesized data is translated along $\mathrm{x}, \mathrm{z}$ axis and rotated around $\mathrm{x}$ axis, respectively, as the difference between the ground truth and the estimated parameters. When the initial translation/rotation amount is set as shown in the translation/rotation axis $(0.01 \mathrm{x}-\mathrm{t}, 0.02$ $\mathrm{x}$-t, $0.03 \mathrm{x}-\mathrm{t}$ in the left graph), the difference between each estimated parameter in the parameter axis (a, b, scale) and its ground truth is shown in a vertical axis, $(0.00, \pm 0.02, \pm$ 0.04 in the left graph).

$\mathrm{Z}$ axis for this surface is the direction of expanding/contracting. The translation along the $\mathrm{z}$ axis results in the ambiguity of the parameter estimation. In contrast, the translation along the $\mathrm{x}$ axis is not directly related to the expansion and contraction. The translation along $\mathrm{z}$ axis simultaneously affects the translation/rotation and deformation parameters. In fact, more iteration was needed for good estimation in case of the translation along the $\mathrm{z}$ axis than in case of the translation along and rotation around the $\mathrm{x}$ axis.

The final error we examined was the result of the initial deformation parameter. The synthesized data were made in the same manner, and the standard deviation of the noise ( $\sigma$ ) was set to 0.0004 . The initial pose and position of the synthesized data were set to the same as those for the computed data. Effects of each initial deformation parameter was investigated, and each initial parameter was changed incrementaly from the ground truth.

The results of estimation are shown in Figure 8 . In the figure, the left, middle and right graph shows the estimation result in the case where the $a, b$ and $l$ are set to each value shown in the horizontal axis of the graph, respectively, as the difference between the ground truth and the estimated parameters. When the initial deformation parameter is set as shown in the registration axis (for example, $0.07,0.06$ , $0.04,0.03$ in the left graph), the difference between each estimated parameter in the parameter axis (a, b, scale) and its ground truth is shown in the vertical axis (for example, $0.000, \pm 0.005, \ldots$, in the left graph). Figure 8 indicates 


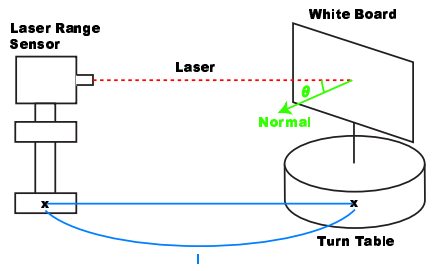

Figure 4. System to evaluate measurement error. In this system, the surface normal and the distance from the white board to the laser range sensor are changeable.

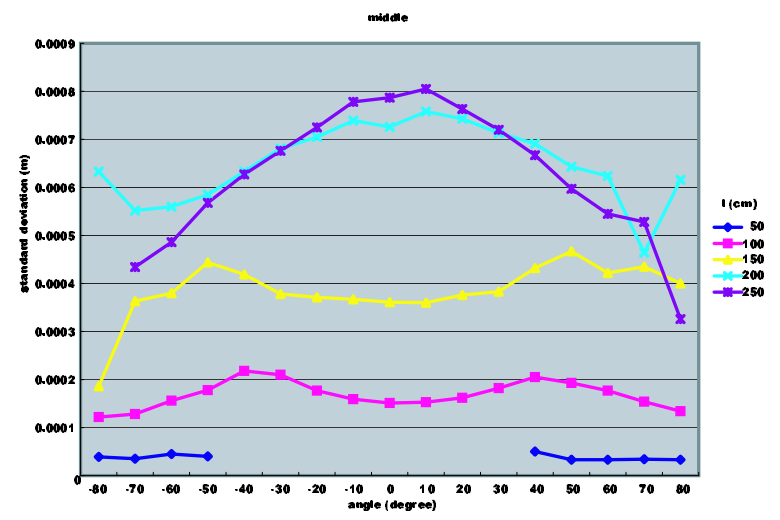

Figure 5. The relationship between surface normal and standard deviation. Each curve showed the data at different distance from white board to the finder.

that the effect of incorrect initial value on each parameter was different. It is difficult to recognize the accuracy of our algorithm via the numerical result, but the registration result is visually almost the same as shown in Figure 3 .

\section{Inter-and-Intra Scanning Registration}

In order to measure large objects effectively, we have developed a novel 3D measurement system: the Floating Laser Range Sensor (FLRS). FLRS digitizes objects from the air while being suspended beneath a balloon platform.

Several conventional aerial measurement systems have been proposed in the past. For example, aerial 3D measurements have been achieved with a laser range sensor installed on a helicopter platform [17] [18] . High frequency vibration of the platform, however, must be considered in obtaining highly accurate results. Another technique is aerial stereo photography with a digital camera that is attached to a balloon [19] ; however, this stereo method cannot achieve a satisfactory level of precision in the restored data.

To overcome these difficulties, we designed FLRS. It is free from high frequency vibration such as that of a helicopter engine; there still remains low frequency movement due to the floating balloon, causing distortion in obtained data. This movement is generated by

- Initial velocity
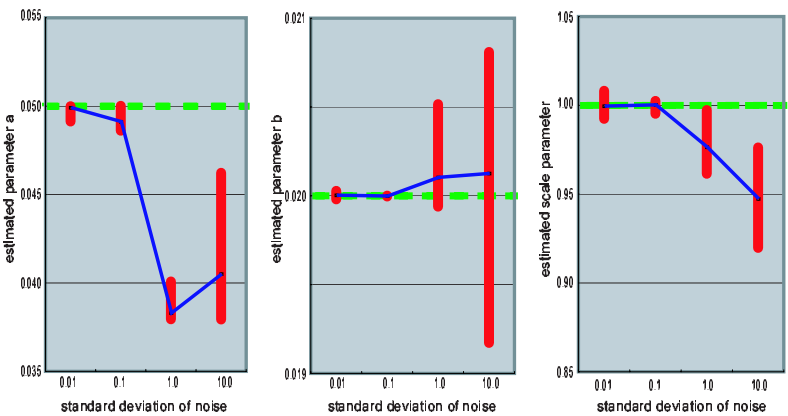

Figure 6. The maximum and the minimum of estimated parameter.
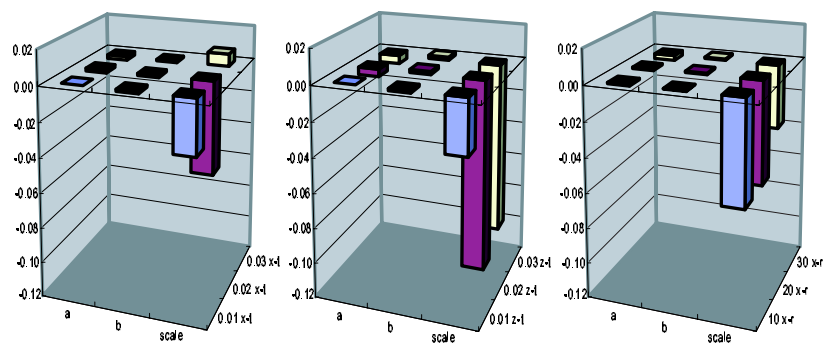

Figure 7. Estimation result in each case of initial translation and rotation error.

- Initial angular velocity

- Acceleration generated by external force

- Angular acceleration generated by external moment

We can ignore the influence of translation and angular acceleration because FLRS needs only one second to scan each frame. And insignificant rotation can be approximated to translation movement. Hence we consider only constant velocity movement. Under this assumption, we set up the deformation equation in Equation ( 8 ).

In this case, the geometrical function $\mathbf{g}\left(\mathbf{x}_{i}, \mathbf{k}\right)$ is represented only by constant velocity vector $\mathbf{v}$ of FLRS movement, and Equation ( 8 ) is replaced with:

$$
\mathbf{g}\left(\mathbf{x}_{i}, \mathbf{v}\right)=\mathbf{x}_{i}-\tau_{i} \mathbf{v}
$$

where $\tau_{i}$ is $i$ th point's captured time passed since the start of the scanning. The descent gradient is represented in this case as follows.

$$
\begin{gathered}
\frac{\partial z_{i j}(\mathbf{p})}{\partial \mathbf{p}}=\left[\begin{array}{c}
2\left(\mathbf{g}\left(\mathbf{x}_{i}, \mathbf{v}\right)+\mathbf{t}-\mathbf{y}_{j i}\right) \\
-4 \mathbf{g}\left(\mathbf{x}_{i}, \mathbf{v}\right) \times\left(\mathbf{t}-\mathbf{y}_{j i}\right) \\
2\left(\mathbf{g}\left(\mathbf{x}_{i}, \mathbf{v}\right)+\mathbf{t}-\mathbf{y}_{j i}\right) \frac{\partial \mathbf{g}\left(\mathbf{x}_{i}, \mathbf{v}\right)}{\partial \mathbf{v}}
\end{array}\right], \\
\text { where } \quad \mathbf{p}=\quad(\mathbf{t}, \mathbf{q}, \mathbf{v}), \\
\frac{\partial \mathbf{g}\left(\mathbf{x}_{i}, \mathbf{v}\right)}{\partial \mathbf{v}}=\left[\begin{array}{ccc}
\left(\tau_{i}\right. & 0 & 0)^{T} \\
(0 & \tau_{i} & 0)^{T} \\
(0 & 0 & \left.\tau_{i}\right)^{T}
\end{array}\right] .
\end{gathered}
$$

While translation and rotation registration are due to the sensor movement among multiple views, the shape deformation 

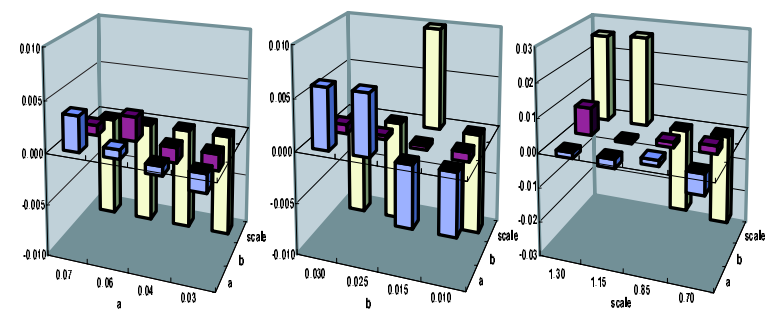

Figure 8. Estimation result in each case of an initially set deformation parameter.
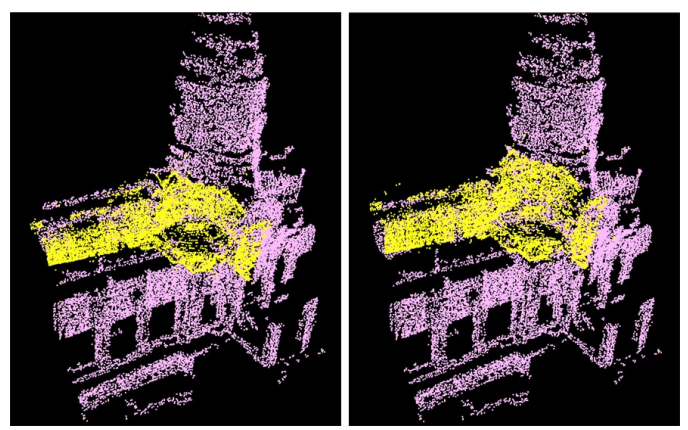

Figure 9. Range images before and after our registration process: The left image shows the data under the initially set translation, rotation, and deformation parameters. A range image of FLRS (yellow) is aligned and fitted onto the corresponding range image of Cyrax 2500 (purple) simultaneously as shown in the right image. These range images are the partial shape of the Bayon temple in Cambodia.

registration is due to the sensor movement during one scan. Thus, we refer to this registration as "inter-and-intra scanning registration".

\subsection{Experiment}

As an experiment on an actual case, we executed our algorithm against the data of the Bayon temple. In this experiment, we aligned the corresponding data captured by FLRS and Cyrax 2500. The latter data was scanned from stable ground, so there was no movement during scanning, and we assume that it is sufficiently reliable. The result is shown in Figure 9 . This figure shows that our algorithm aligned and fitted the FLRS's data well onto the Cyrax2500's data.

\subsection{Evaluation}

To evaluate the accuracy of the algorithm, we aligned the original and synthesized data through our algorithm. The synthesized data translates, rotates, and distorts the original data with known parameters. The optimal deformation registration parameters between the original and synthesized data are the known parameters. We investigated how close the parameter obtained through our algorithm was to the known

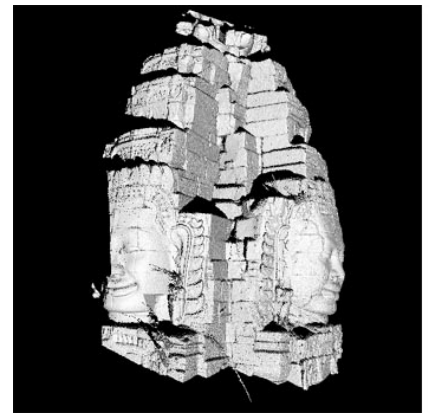

(a)

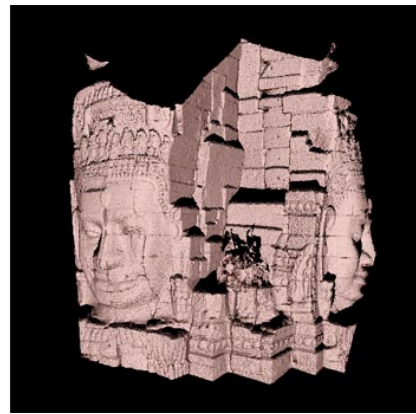

(b)
Figure 10. Sample range images for evaluation experiments.

Table 1. The setting difference between the original and the transformed in each parameter for the accuracy evaluation of the deformation registration.

\begin{tabular}{c|c}
\hline \hline parameter & setting difference \\
\hline $\mathbf{R}(\mathbf{q})$ & $3[\mathrm{deg}]$ around $X$ axis \\
\hline $\mathbf{t}$ & $0.1[\mathrm{~m}]$ along $X$ axis \\
$\mathbf{v}$ & $\begin{array}{r}0.00-3.00[\mathrm{~m} / \mathrm{s}] \text { along } X \text { axis } \\
\text { (every } 0.01[\mathrm{~m} / \mathrm{s}] \text { increment) }\end{array}$ \\
\hline \hline
\end{tabular}

parameter. The synthesized data are created from a range image by removing points randomly to make them sparser and to prevent the same points from being included in both sets of data, in order to simulate actual conditions in the registration of the images at different sites. As with conventional registration, each initial parameter was set via GUI, and then our algorithm was executed.

We investigated the difference between the parameter obtained by our algorithm and the known parameter according to the change of $\mathbf{v}$ in the condition shown in Table 1 . The deformation registration was executed five times at each setting parameter of $\mathbf{v}$ in order to remove the outlier, and the difference was calculated as the average. The result is shown in Figure 11.

The difference is stably constant in each parameter in the condition where the setting velocity is within $1.6[\mathrm{~m} / \mathrm{s}]$, but is drastically oscillated otherwise. This result led us to conclude that our algorithm can obtain an accurate deformation parameter for distortion correction as long as the sensor velocity is within $1.6[\mathrm{~m} / \mathrm{s}]$.

Next, we focused on the estimation accuracy of the obtained parameter under $1.6[\mathrm{~m} / \mathrm{s}]$ of the sensor velocity. The average difference of $\mathbf{t}, \mathbf{R}$, and $\mathbf{v}$ is $0.005[\mathrm{~m}], 0.1[\mathrm{deg}]$, and $0.008[\mathrm{~m} / \mathrm{s}]$ respectively. Note that sizes of faces in the images are over 1 meter. 


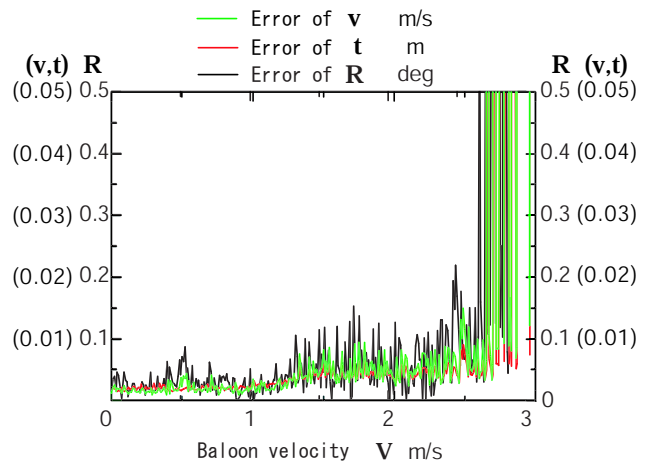

Figure 11. The difference from the optimum $(\mathrm{v}, \mathrm{t}, \mathrm{R})$

\section{Conclusion and Future Work}

We proposed an extended registration framework which allows 3D data to be deformed. Our proposed method assumes that the deformation is strictly defined by some parameterized fomulation derived from the deformation mechanism. Deformation registration can be reduced to a problem of minimizing the error function, which is the squaered sum of the distance between the corresponding points in the data. While conventional registration methods minimize this error function concerned about six parameters (three translation parameters and three rotation parameters for rotation), the error function in our proposed framework includes the deformation parameters as well.

We introduced two applications in this paper. One is shape parameter estimation, and the other is shape rectification. In the first application, accurate shape parameters for the mathematical model could be estimated. The other application rectified the distorted data obtained from the Floating Laser Range Sensor (FLRS) which was suspended under a balloon platform. And the estimation accuracy was also shown in each application. The deformation parameter estimation is the mean of the 3D data fitting. The objective was also to understand the cause and origination of the deformation, so these can be used for system feedback.

Our applications are only a few of the possible applications, and we are trying to develop an application to generate the CAD primitives under the shape parameter estimated from range images. This application will convert the range images into the properly approximated CAD data. The benefit of this application is to be able to compress the range images, which usually consist of numerous 3D points and polygons. We intend to apply our framework widely to various class of problem in the future. This work was supported in part by Japan Science and Technology Cooperation (JST) under the CREST program. Scanning the Bayon temple was conducted jointly with Japanese Government Team for Safeguarding Angkor (JSA), the University of Tokyo and JST.

\section{References}

[1] C. V. Stewart, C. L. Tsai, and A. Perera. A view-based approach to registration: Theory and application to vas- cular image regisrtaion. In Proceedings of International Conference on Information Processing in Medical Imaging (IPMI), pages 475-486, 2003.

[2] J. and N. Ayache. Rigid and affine registration of smooth surfaces using differential properties. In Proceedings of Third European Conference on Computer Vision (ECCV'94), pages 397-406, 1994.

[3] A. Guéziec, X. Pennec, and N. Ayache. Medical image registration using geometric hashing. 4(4):29-41, 1997.

[4] Eric Bardinet, Laurent D. Cohen, and Nicholas Ayache. A parametric deformable model to fit unstructured $3 \mathrm{~d}$ data. 71(1):39-54, 1998.

[5] P. R. Andresen and M. Nielsen. Non-rigid registration by geometryconstrained diffusion. In Proceedings of Medical Image Computing and Computer-Assisted Intervention (MICCAI'99), pages 533-543, 1999.

[6] P.J. Besl and N.D. McKay. A method for registration of 3-d shapes. IEEE Transactions on Pattern Analysis and Machine Intelligence, 14(2):239-256, February 1992.

[7] G. Turk and M. Levoy. Zipped polygon meshes from range images. In ACM SIGGRAPH Proceedings, pages 311-318, July 1994.

[8] K. Higuchi, M. Herbert, and K. Ikeuchi. Building 3-d models from unregistered range images. In Graphical Models and Image Processing, volume 57, pages 315333, July 1995.

[9] A.E. Johnson and M. Herbert. Surface matching for object recognition in complex 3-dimensional scenes. Image and Vision Computing, 16(9/10):635-651, July 1998.

[10] P.J. Besl and N.D. McKay. A method for registration of 3-d shapes. IEEE Transactions on Pattern Analysis and Machine Intelligence, 14(2):239-256, February 1992.

[11] David Simon. Fast and Accurate Shape-Based Registration. PhD thesis, School of Computer Science, Carnegie Mellon University, 1996.

[12] Szymon Rusinkiewicz and Marc Levoy. Efficient varinats of the icp algorithm. In Proceedings of the 3rd International Conference on 3D Digital Imaging and Modeling, pages 145-152, May 2001.

[13] Y. Chen and G.G. Medioni. Object modeling by registration of multiple range images. Image and Vision Computing, 10(3):145-155, 1992.

[14] P. Neugebauer. Geometrical cloning of 3d objects via simultaneous registration of multiple range images. In Proceedings of International Conference on Shape Modeling and Application, pages 130-139, March 1997.

[15] A.E. Johnson and S. Kang. Registration and integration of textured 3-d data. In Proceedings of International Conference on 3D Digital Imaging and Modeling, pages 234-241, May 1997. 
[16] Mark D. Wheeler. Automatic Modeling and Localization for Object Recognition. PhD thesis, School of Computer Science, Carnegie Mellon University, 1996.

[17] Mark Daniel Sebastian Thrun and Dirk H ahnel. Scan alignment and 3-d surface modelling with a helicopter platform. In The 4th Int. Conf. on Field and Service Robotics, July 14-16, 2003.

[18] Ryan Miller and Omead Amidi. 3-d site mapping with the cmu autonomous helicopter. June 1998.

[19] Jana Visnovcova, Li Zhang, and Armin Gruen. Generating a $3 \mathrm{~d}$ model of a bayon tower using non-metric imagery. In Proc. of Int. Workshop Recreating the Past -Visualization and Animation of Cultural Heritage, 2001. 